\title{
Top quark pair production cross section measurements with the ATLAS detector
}

\author{
A. Hasib*t \\ University of Oklahoma \\ E-mail: a.hasibecern.ch
}

\begin{abstract}
Measurements of the inclusive and the differential top quark pair production cross sections in proton-proton collisions at the center of mass energy of 8 and $13 \mathrm{TeV}$ with the ATLAS detector at the Large Hadron Collider are presented. The inclusive measurements reach high precision and are compared to the best available theoretical calculations. Differential measurements of the kinematic properties of the top quark production are also discussed. These measurements, including results using boosted top quarks, probe our understanding of top quark pair production in the $\mathrm{TeV}$ regime. The results, unfolded to particle and parton level, are compared to Monte Carlo generators implementing LO and NLO matrix elements matched with parton showers and NNLO QCD calculations.
\end{abstract}

XXIV International Workshop on Deep-Inelastic Scattering and Related Subjects

11-15 April, 2016

DESY Hamburg, Germany

\footnotetext{
* Speaker.

${ }^{\dagger}$ On behalf of the ATLAS collaboration.
} 


\section{Introduction}

The Large Hadron Collider [1] is a top quark factory and allows for the precise measurement of the inclusive, as well as, the differential top quark pair $(t \bar{t})$ production cross section. All properties of the top quark, with the exception of its mass, are predicted by the Standard Model (SM). The inclusive cross section, $\sigma_{t \bar{t}}$, measured in different final states, provides a check of consistency with each other and with the prediction of the SM. Any inconsistency or deviation from the prediction of the SM would be an evidence of new physics. The differential cross section provides additional constraint on the fundamental parameters of the SM and can be used to tune Monte Carlo event generators. At the LHC energy regime, the dominant production mechanism ( $\sim 87 \%)$ of top quark pairs is gluon-gluon fusion and quark anti-quark interactions are responsible for the rest of the production. According to the SM, the top quark decays over $99 \%$ of the time to a $W$ boson and a $b$-quark. The different final states of the $W$ boson determines the different $t \bar{t}$ final states. Measurements of the cross section in different final states really are measurements of $\sigma_{t \bar{t}} \cdot B R_{1} \cdot B R_{2}$, where, $B R_{1}$ and $B R_{2}$ are the branching ratios of the top and the anti-top quark, respectively. To determine the inclusive cross section, the measurements assume the branching ratios to different final states are determined by the $W$ boson branching ratios. The inclusive cross section measured using the data collected by the ATLAS [2] detector at $\sqrt{s}=13 \mathrm{TeV}$ is presented here for the following final states:

- $e \mu+b$-jet: exactly one isolated electron $\left(p_{T}^{e}>25 \mathrm{GeV}\right)$ and one isolated muon $\left(p_{T}^{\mu}>25 \mathrm{GeV}\right)$ with opposite electric charge. The electrons (muons) are required to be isolated in the calorimeter using the energy deposition, $E_{T}$, inside a cone of $\Delta R<0.2$, where, the distance in the $\eta-\phi$ space is defined as $\Delta R=\sqrt{(\Delta \eta)^{2}+(\Delta \phi)^{2}}$. A track isolation requirement is also applied requiring the electron (muon) tracks to be isolated in a cone which increases in size, $\Delta R=10 \mathrm{GeV} / p_{T}^{e / \mu}$, for decreasing $p_{T}$ up to a maximum of $\Delta R=0.2$. The electron (muon) identification efficiency is $90 \%$ for $p_{T}$ of $25 \mathrm{GeV}$, which increases to $99 \%$ at $60 \mathrm{GeV}$ and is measured in $Z \rightarrow e e(Z \rightarrow \mu \mu)$ events. In addition, a requirement of at least one $b$-tagged jet with efficiency about $70 \%$ is applied to select events in this final state.

- $e e / \mu \mu+b$-jet: exactly two isolated electrons or two isolated muons with opposite electric charge, at least one $b$-jet. The selection criteria of electrons and muons are same as the $e \mu+b$-jet final state. Additionally, the invariant mass $\left(m_{\ell \ell}\right)$ of the two electrons (muons) are required to be above $60 \mathrm{GeV}$ with a veto around the $Z$ boson mass, $81<m_{\ell \ell}<101 \mathrm{GeV}$.

- $e / \mu+$ jets: exactly one isolated electron or muon, at least four jets (anti- $k_{t}$ algorithm, $R=0.4$, $p_{T}^{j}>25 \mathrm{GeV}$ ), at least one of which is a $b$-jet. Additional requirements on the missing transverse momentum, $E_{T}^{\text {miss }}$, and the $W$ boson transverse mass, $m_{T}^{W}=\sqrt{2 p_{T}^{\ell} E_{T}^{\text {miss }}(1-\cos \Delta \phi)}$ are applied to reduce the fake (misidentified) lepton background. In electron channel a requirement of $E_{T}^{\text {miss }}>40 \mathrm{GeV}$ or $m_{T}^{W}>50 \mathrm{GeV}$ is applied, whereas, in the muon channel a requirement of $E_{T}^{\text {miss }}+m_{T}^{W}>60 \mathrm{GeV}$ is applied.

The differential cross sections are measured using the $e / \mu+$ jets final state at $\sqrt{s}=8 \mathrm{TeV}$. All the measurements assume the top quark mass to be $172.5 \mathrm{GeV}$. 


\section{Inclusive cross section measurements}

The inclusive cross section in $e \mu+b$-jet final state is calculated at $\sqrt{s}=13 \mathrm{TeV}$ using the full 2015 dataset corresponding to $\int \mathscr{L} d t=3.2 \mathrm{fb}^{-1}$ [3]. The analysis extracts the $\sigma_{t \bar{t}}$ by counting the number of events with exactly one $\left(N_{1}\right)$ and exactly two $\left(N_{2}\right)$ b-tagged events expressed as,

$$
N_{1}=L \sigma_{t \bar{t}} \varepsilon_{e \mu} 2 \varepsilon_{b}\left(1-C_{b} \varepsilon_{b}\right)+N_{1}^{b k g} \quad \text { and } \quad N_{2}=L \sigma_{t \bar{t}} C_{b} \varepsilon_{e \mu} \varepsilon_{b}^{2}+N_{2}^{b k g},
$$

where, $\varepsilon_{e \mu}$ is the $e \mu$ efficiency, $\varepsilon_{b}$ is the $b$-tagging efficiency and $C_{b}$ is the tagging correlation coefficient close to unity. A likelihood fit is then used to simultaneously determine the cross section and the $b$-tag efficiency. The purity of the signal events in the $1 b$-tag ( $2 b$-tag) region is $89 \%$ (96\%). The largest contribution in the background processes comes from the single top $W t$ channel and is normalized to the approximate NNLO cross section of $71.7 \pm 3.8 \mathrm{pb}$, determined as in Ref. [5]. The $Z+$ jets background is determined by measuring the rates of $Z \rightarrow e e$ and $Z \rightarrow \mu \mu$ events with one and two $b$-tagged jets in both data and simulation, and using the resultant ratio to scale the simulation estimate of background from $Z \rightarrow \tau \tau+$ jets $\rightarrow e \mu+$ jets. The background originating from a misidentified electron or muon is estimated in data events using $e \mu$ events having the electric charge. In addition to the inclusive $\sigma_{t \bar{t}}$, the cross section is also measured in a fiducial volume. The measured cross sections are,

$$
\begin{aligned}
& \left.\left.\sigma_{t \bar{t}}^{\text {inclusive }}=803 \pm 7 \text { (stat. }\right) \pm 27 \text { (syst.) } \pm 45 \text { (lumi. }\right) \pm 12 \text { (beam) pb } \\
& \left.\left.\sigma_{t \bar{t}}^{\text {fiducial }}=11.12 \pm 0.10 \text { (stat. }\right) \pm 0.28 \text { (syst.) } \pm 0.62 \text { (lumi. }\right) \pm 0.17 \text { (beam) pb. }
\end{aligned}
$$

The uncertainty on the luminosity (5.5\%) and the beam energy (1.5\%) significantly limits the precision of this measurement. The systematic uncertainty from experimental sources is small $(1.5 \%)$ compared to the theoretical sources $(3 \%)$. The cross section is thus measured also in the fiducial volume which reduces the theoretical sources of uncertainty to $2 \%$. The results of this measurement have since been updated, see Ref. [4].

The $e e / \mu \mu+b$-tag final state uses the same method as the $e \mu+b$-tag final state to extract the cross section. The analysis uses dataset corresponding to $\int \mathscr{L} d t=85 \mathrm{pb}^{-1}$ at $\sqrt{s}=13 \mathrm{TeV}$ [6]. The backgrounds are estimated using the same method as that of the $e \mu+b$-tag analysis. The measured inclusive cross section is,

$$
\left.\sigma_{t \bar{t}}^{\text {inclusive }}=749 \pm 57 \text { (stat. }\right) \pm 79 \text { (syst.) } \pm 74 \text { (lumi.). }
$$

The cross section in the $e / \mu+$ jets final state is also measured at $\sqrt{s}=13 \mathrm{TeV}$ with an integrated luminosity of $\int \mathscr{L} d t=85 \mathrm{pb}^{-1}$ [6]. The dominant background in this final state comes from $W+$ jets processes which is measured in data using the charge asymmetric production of the $W$ boson in $p p$ collisions [7]. The misidentified lepton background is also measured in data that relies on a relaxed lepton identification criteria. The cross section is measured by counting the number of signal events in data. The measured cross section is,

$$
\sigma_{t \bar{t}}^{\text {inclusive }}=817 \pm 13 \text { (stat.) } \pm 103 \text { (syst.) } \pm 88 \text { (lumi.). }
$$

The precision of the cross section measurements presented above suffers from large uncertainty on the integrated luminosity. This uncertainty largely cancels in the measurement of the 
ratio of the inclusive $t \bar{t}$ to $Z$ boson cross sections, $R_{t \bar{t} / Z}$ [6]. This measurement provides significant constraint on the ratio of gluon to sea-quark content in the proton parton distribution functions (PDF) [8]. The $\sigma_{t \bar{t}}$ is measured in the $e \mu$ final state described in Ref. [9] and uses an integrated luminosity of $78 \mathrm{pb}^{-1}$. The $Z$ boson cross section measurement is described in Ref. [10] and uses $85 \mathrm{pb}^{-1}$ of data. The updated results for both these measurements are in Refs. [4, 11]. The ratio of the two cross sections is measured to be,

$$
R_{t \bar{t} / Z}=0.445 \pm 0.027 \text { (stat.) } \pm 0.028 \text { (syst.) }
$$

The measured ratio is compared to the theoretical predictions calculated at NNLO in QCD using CT10nnlo [12], ABM12LHC [15], NNPDF3.0 [14] and MMHT14nnlo68CL [13] PDF sets. Figure 1 shows the comparison of the theoretical predictions calculated with these PDF sets to the measured ratio. The experimental result is consistent with the theoretical predictions calculated using CT10, NNPDF3.0 and MMHT14nnlo68CL PDF sets. The ABM12LHC PDF is, however, only marginally consistent as it predicts $12 \%$ smaller cross section for $t \bar{t}$ compared to CT10 PDF.

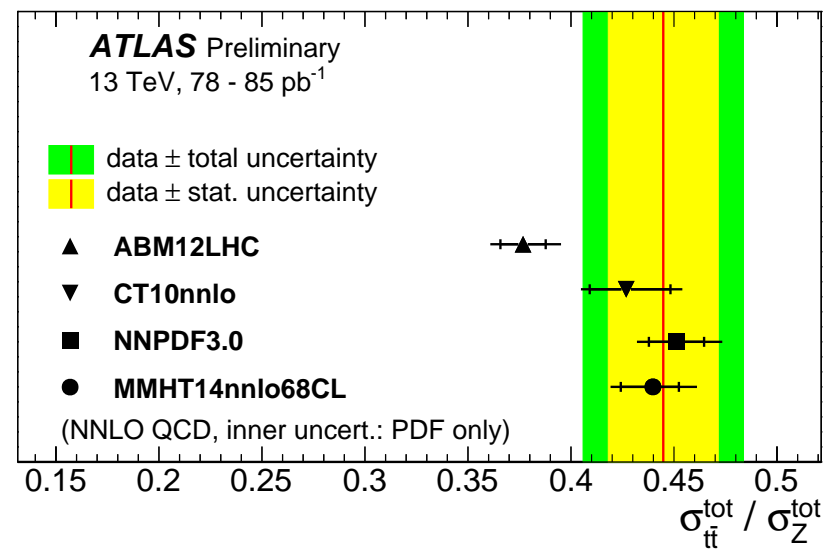

Figure 1: Measured cross section ratio $R_{t \bar{t} / Z}$ compared to NNLO predictions at $\sqrt{s}=13 \mathrm{TeV}$ based on the ABM12LHC, CT10, NNPDF3.0 and MMHT14 PDF sets [6].

\section{Differential cross section measurements}

The differential cross section measurements can be used to study the characteristics of $t \bar{t}$ production with respect to different observables that are sensitive to the PDF, initial and final state radiation, non-resonant processes and higher order corrections. In addition, it can be sensitive to beyond SM processes that manifest as modifications of $t \bar{t}$ differential distributions with respect to the SM predictions. The differential cross sections presented here, are measured in the $e / \mu+\mathrm{jets}$ final state in the resolved and the boosted topology at $\sqrt{s}=8 \mathrm{TeV}$ using dataset corresponding to $\int \mathscr{L} d t=20.3 \mathrm{fb}^{-1}[16,17]$. In the resolved topology, the top quark is produced almost at rest and is characterized by isolated leptons and jets with small $\Delta R(=0.2)$. In the boosted topology, the high $p_{T}(>300 \mathrm{GeV})$ jets will tend to be collimated and merged with the $W$ boson resulting into large $\Delta R(=1.0)$ jets. 
The differential cross sections are measured as a function of the top quark transverse momentum $\left(p_{T}^{t}\right)$ and transverse momentum $\left(p_{T}^{t \bar{t}}\right)$, rapidity $\left(y^{t \bar{t}}\right)$, invariant mass $\left(m^{t \bar{t}}\right)$ of the $t \bar{t}$ system. The observables are corrected for detector effects to the parton level with extrapolation to full phase space and compared to those predicted using three different generators: POWHEG, MC@NLO and MadGraph. Figure 2 shows these comparisons in the resolved topology. POWHEG+PYTHIA with CT10 PDF and the $h_{\text {damp }}$ parameter that regulates the high- $p_{T}$ radiation, set to the top quark mass ( $m_{\text {top }}$ ) provides the best description of $d \sigma^{t \bar{t}} / d p_{T}^{t \bar{t}}$ and $d \sigma^{t \bar{t}} / d m^{t \bar{t}}$. None of the generators provide a good description of the $\left|y^{t \bar{t}}\right|$, especially in the forward region. For low $p_{T}^{t}$, most of the generators provide a good description, however for $p_{T}>250 \mathrm{GeV}$, the prediction in simulation is harder compared to that observed in the data events. In the boosted topology, a similar trend in simulated $p_{T}^{t}$ spectrum is observed for different generators and PDF sets as shown in Fig. 3. The HERAPDF [18] along with POWHEG+PYTHIA $\left(h_{\text {damp }}=m_{\text {top }}\right)$ provides the closest description in the boosted topology. In the resolved topology, the predictions are also calculated at approximate next-to-next-to-next-to-leading order ( $\mathrm{aN}^{3} \mathrm{LO}$ ), aNNLO and full NNLO orders in QCD. A full NNLO calculation is found to provide a better description compared to the higher order approximate calculations. The observables are also corrected to the particle level in a fiducial volume, which shows similar trends as observed at the parton level in the full phase space. The uncertainties at the fiducial level is smaller compared to the full phase space but results in the reduction of the agreement between data and simulation.

\section{Conclusion}

The inclusive cross section measurements in $p p$ collisions at $\sqrt{s}=13 \mathrm{TeV}$ using the ATLAS detector is presented. The measurements are in excellent agreement with the SM prediction of $832 \pm 42 \mathrm{pb}$ calculated at next-to-next-to-leading order (NNLO) plus next-to-next-to-leadinglogarithm (NNL) [19]. All the measurements suffer from large luminosity uncertainty. The $e \mu+b-$ jet final state provides the most precise measurement with a total uncertainty of $6.7 \%$ where the experimental accuracy is better than the theoretical precision. The differential cross section is measured at $\sqrt{s}=8 \mathrm{TeV}$ in the $e / \mu+$ jets final state for both resolved and boosted topology. All generators predict harder $d \sigma_{t \bar{t}} / d p_{T}^{t}$ than observed with the full NNLO calculation describing the data better than approximate higher order QCD calculations.

\section{References}

[1] E. Lyndon and B. Phillip (Eds.), JINST 03 (2008) S08001.

[2] ATLAS Collaboration, JINST 03 (2008) S08003.

[3] ATLAS Collaboration, ATLAS-CONF-2016-005.

[4] ATLAS Collaboration, CERN-EP-2016-088 [hep-ex/1606.02699].

[5] N. Kidonakis, Phys. Rev. D 82 (2010) 054018.

[6] ATLAS Collaboration, ATLAS-CONF-2015-049.

[7] F. Halzen, Y. S. Jeong, and C. S. Kim, Phys. Rev. D 88 (2013) 073013. 

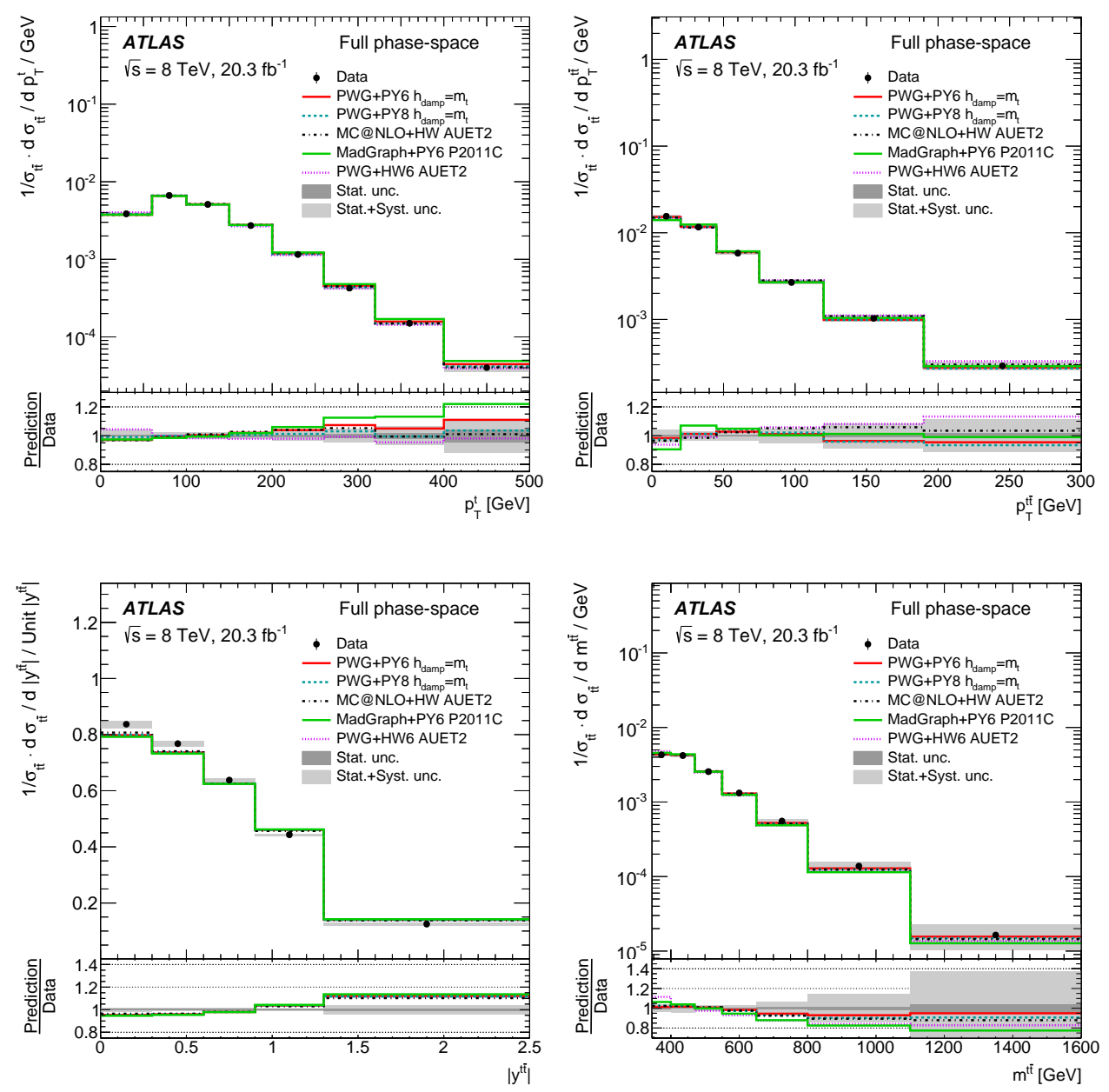

Figure 2: Normalized differential cross section as a function of $p_{T}^{t}, p_{T}^{t \bar{t}},\left|y^{t \bar{t}}\right|$ and $m^{t \bar{t}}$ in the resolved topology [16].
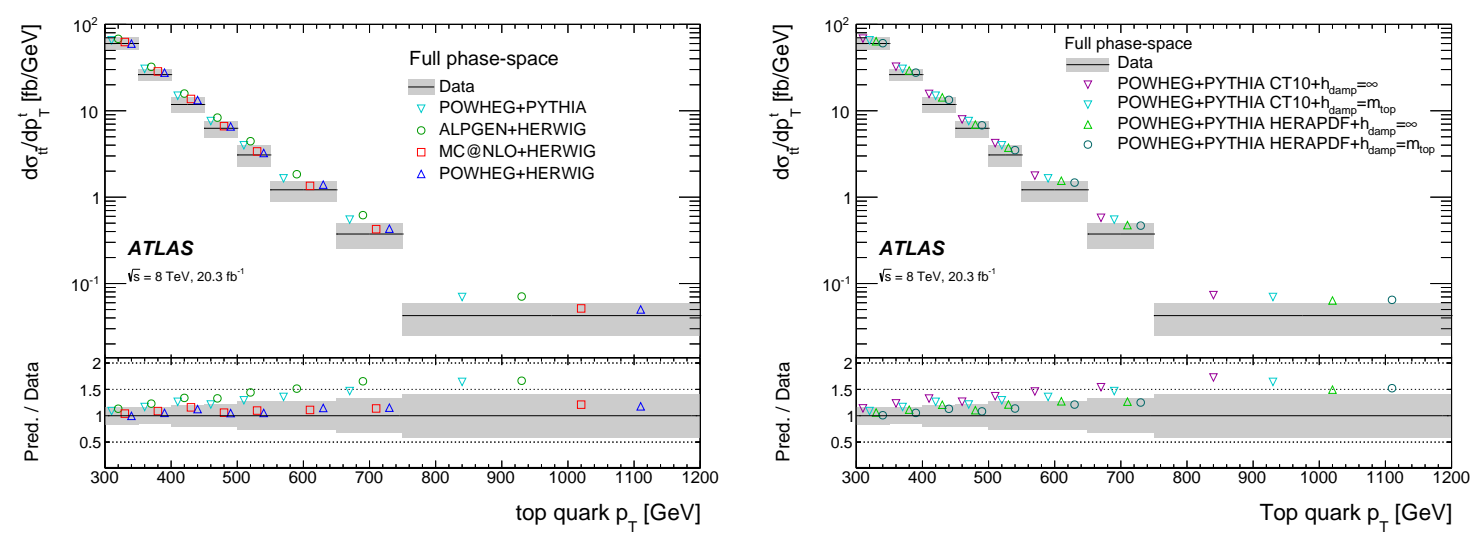

Figure 3: Differential cross section as a function of $p_{T}^{t}$ in the boosted topology compared to different generators (left) and different PDF sets (right) [17]. 
[8] J. Rojo et al., J.Phys. G42 (2015) 103103.

[9] ATLAS Collaboration, ATLAS-CONF-2015-033.

[10] ATLAS Collaboration, ATLAS-CONF-2015-039.

[11] ATLAS Collaboration, CERN-EP-2016-069 [hep-ex/1603.09222].

[12] J. Gao et al., Phys. Rev. D 89 (2014) 033009.

[13] L. A. Harland-Lang, A. D. Martin, P. Motylinski, and R. S. Throne, Eur. Phys. J. C75 (2015) 204.

[14] NNPDF Collaboration, R. D. Ball et al., JHEP 04 (2015) 040.

[15] S. Alekhin, J. Blümlein, and S. Moch, Phys. Rev. D 89 (2014) 054028.

[16] ATLAS Collaboration, CERN-PH-EP-2015-239.

[17] ATLAS Collaboration, Phys. Rev. D 93 (2016) 032009.

[18] H1 and Zeus Collaboration, JHEP 01 (2010) 109.

[19] M. Czakon and A. Mitov, Comp. Phys. Comm. 185 (2014) 2930. 